\title{
Association between a prolonged corrected QT interval and outcomes in patients in a medical Intensive Care Unit
}

\author{
Tarun K. George, David Chase ${ }^{1}$, John Victor Peter², Sowmya Satyendra, R. Kavitha ${ }^{3}$, \\ Leah Raju George ${ }^{4}$, Vineeth Varghese Thomas ${ }^{2}$
}

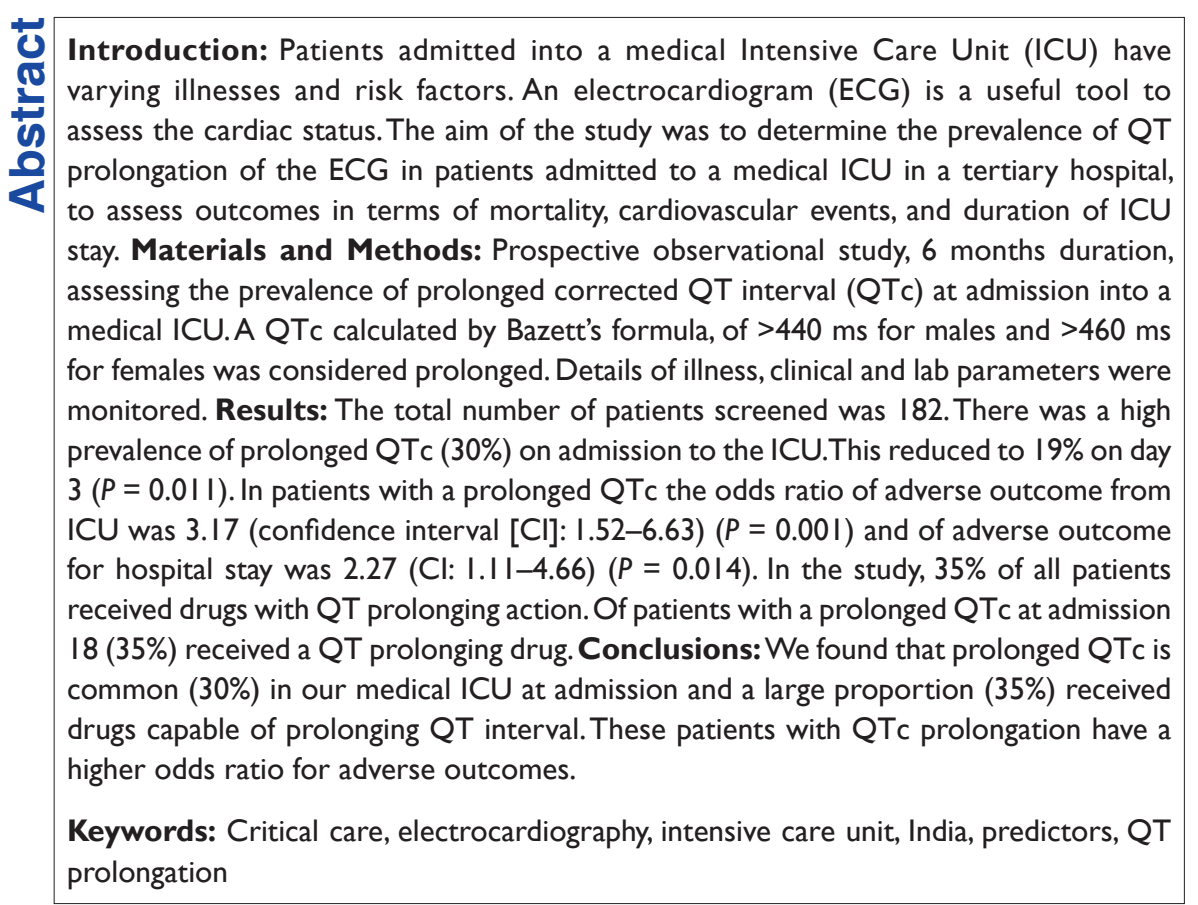

\begin{tabular}{|l|}
\hline Access this article online \\
\hline Website: www.jiccm.org \\
\hline DOI: 10.4103/0972-5229.158271 \\
\hline Quick Response Code: \\
\hline \\
\hline
\end{tabular}

\section{Introduction}

A medical Intensive Care Unit (ICU) has a wide variety of patients varying in age and primary illness. These patients have a high morbidity and a guarded prognosis. ${ }^{[1]}$ At admission, a thorough clinical and laboratory assessment needs to be made for a clear picture of the diagnosis, severity of the illness,

\section{From:}

Departments of Medicine, ${ }^{1}$ Cardiology Electrophysiology, ${ }^{2}$ Critical Care Medicine, ${ }^{3}$ Biostatistics and ${ }^{4}$ Anaesthesiology, Christian Medical College,

Vellore, Tamil Nadu, India

\section{Correspondence:}

Dr. Tarun K George, Department of Medicine, Christian Medical College, Vellore - 632 004, Tamil Nadu, India.

E-mail: tarunkg@gmail.com appropriate supportive, and specific therapy. This also helps to prognosticate the course and outcome. There are numerous scoring systems that are routinely employed especially the Acute Physiology and Chronic Health Evaluation (APACHE), Simplified Acute Physiology

This is an open access article distributed under the terms of the Creative Commons Attribution-NonCommercial-ShareAlike 3.0 License, which allows others to remix, tweak, and build upon the work non-commercially, as long as the author is credited and the new creations are licensed under the identical terms. For reprints contact: reprints@medknow.com

How to cite this article: George TK, Chase D, Peter JV, Satyendra S, Kavitha R, George LR, Thomas VV. Association between a prolonged corrected QT interval and outcomes in patients in a medical Intensive Care Unit. Indian J Crit Care Med 2015;19:326-32. 
Score and Sequential Organ Failure Assessment (SOFA) scores. ${ }^{[2-4]}$ However, these have many variables and do not utilize the electrocardiogram (ECG) among its parameters. An ECG which is taken for all patients in an ICU is a tool which can provide valuable information. The ECG represents the electrical activity of the heart. The QT interval, related to ventricular function, if prolonged has been shown to have associations with poor ICU outcomes. ${ }^{[5]}$ It is seen that in acutely ill patients the prevalence of a prolonged corrected QT interval $(\mathrm{QTc})$ is much higher than one might expect. ${ }^{[6,7]}$ These may be due to an underlying cause which may be correctible. ${ }^{[8]}$ The aim of the study was to determine the prevalence of prolonged QTc at admission and assess associated outcomes in patients admitted to a tertiary care Indian medical ICU.

\section{Materials and Methods}

\section{Setting}

This was a prospective observational study of 6 months duration in a multidisciplinary medical ICU of a tertiary care academic hospital. This was a 24 bed ICU manned by $24 \mathrm{~h}$ in-house doctors and staff comprising of junior and senior level residents, critical care fellows, and specialist intensivists. The period of recruitment was from January to July 2013.

\section{Study participants}

Patients above the age of 18 that were admitted to the medical ICU were included in the study. Patients on temporary or permanent pacemaker, those with WPW pattern on ECG and referrals from other ICUs were excluded. The patients had varied illnesses like sepsis, poisonings, hematological posttransplant patients with opportunistic infections, and patients post-attempted suicide by hanging. The approval of the study was sanctioned by the Institution Review Board. Informed consent was obtained. The patients were recruited at admission and followed-up till discharge.

\section{The outcomes}

The primary outcome to be assessed was the prevalence of a prolonged QTc in the ICU at admission. Secondary outcomes were association of prolonged QTC and duration of ICU and hospital stay, favorable or unfavorable outcome from ICU and hospital, and duration of ventilation free days. A favorable event was discharge from the ICU/hospital and unfavorable was death or discharged against medical advice. The other outcomes assessed were associations of prolonged QTc and arrhythmias (Torsades de Pointes, bradycardia, ventricular tachycardia, ventricular ectopic beats, supraventricular complexes), hypotension, cardiac arrest, acute coronary syndrome, intubation, and death.

We also looked for the prevalence of QT prolonging medication administered to a patient in the first 3 days in ICU.

\section{Methodology}

Patients were admitted into the ICU from either the wards or emergency department. After stabilization, an ECG was taken within $4 \mathrm{~h}$ of admission. If an ECG was available within $3 \mathrm{~h}$ prior to admission from the Causality, it was included. ECGs were taken by trained personnel. The machine for the ECG used was GE Mac 600 using Marquette 12SL ECG analysis program for QT analysis. A data collection Performa containing details of co-morbidities, medications, diagnosis, and need for ICU admission was filled. The relevant blood investigations during the first $12 \mathrm{~h}$ period were documented. The APACHE II and SOFA scores were calculated at admission. The APACHE II score asses the severity of disease at admission utilizing 12 variables with a score upto 71 and correlates with subsequent hospital mortality. It utilizes among clinical variables arterial $\mathrm{PaO}_{2}, \mathrm{pH}$, and electrolytes. The SOFA score comprises of six variables including respiratory, cardiac, hepatic, coagulation, renal, and neurological. Higher scores are associated with increased rate of mortality.

In ICU, the patient underwent continuous heart rate, blood pressure, respiratory rate oxygen saturation, and temperature monitoring. A repeat ECG and blood parameters were noted on day 3 of admission. The collected ECGs of day one and three were scanned printed and analyzed for the QT, RR, and QTc manually. ${ }^{[9,10]}$ The ECGs parameters were calculated at a later date after the patients were not in ICU, in order to prevent an observation bias. The primary investigator underwent a training process from a cardiac electrophysiologist for appropriate calculation of the ECG parameters. The QT interval was calculated by Caliper method. The distance was taken from the beginning of the $Q$ wave and the end to the $T$ was the intersection between a line marked by a tangent to the downslope of $\mathrm{T}$ and the baseline. The machine-generated, RR and QTc interval were noted. The manually calculated values were compared to the automated values in the ECGs. The formula used for calculation of the corrected QT was Bazett's formula. $\mathrm{QTC}=\mathrm{QT} / \sqrt{ } \mathrm{RR}$. A comparison between the investigator and the Cardiologist was also done for the QTc calculation. The medication list on day 1 and day 3 was documented and it was checked for potential QT prolonging drugs. The classification of QT-prolonging drugs and a risk of Torsades de Pointes was obtained from 
lists maintained by the Arizona Center for Education and Research on Therapeutics (www.azcert.org). Drugs with a "known risk" (list 1) a "probable risk" for TdP (list 2) or a "conditional risk" (list 3) were used to characterize the medication list. At discharge, the final diagnosis, duration of ICU and hospital stay, and outcome were noted [Figures 1 and 2]. ${ }^{[11,12]}$

Prolonged QTc for this study was taken as $>440 \mathrm{~ms}$ for males and $>460 \mathrm{~ms}$ for females. ${ }^{[13,14]}$

\section{Statistical methods}

The sample size was calculated based on the primary objective of the study. The primary aim of the study is to find out the prevalence of prolonged QTc. From a previous study, the prevalence of prolonged QTc was found to be $24 \%{ }^{[6]}$ Assuming precision to be \pm 6 for the $95 \%$ confidence interval (CI), the sample size for estimating prevalence was calculated as 203.

For the analysis, QT levels were compared between males and females, different age groups, and other variables using independent samples $t$-test. Associations between normal, prolonged QT intervals with other categorical variables are assessed using Chi-square test. To find the agreement between machine and manual QTS intra class correlation (ICC) was calculated.

\section{Results}

There were totally 182 patients screened. Of this, 2 refused consent, one had a permanent pacemaker, and 5 had poor ECG tracings at admission, hence, the total number included for analysis was 174 [Figure 3].

There were $17 \%$ of the patients with a premorbid cardiac illness prior to admission.

The mean SOFA score $=8.1$ (standard deviation $[\mathrm{SD}]=4.46)$ and $\mathrm{APACHE}=23.3(\mathrm{SD}=8.2)$.

The mean duration of stay in the ICU was 7 days $(\mathrm{SD}=5.8)$; and stay in the hospital was 13 days $(S D=10.1)$. Adverse outcome (which included death and those who were discharged against medical advice) was seen in (61) 35\% of patients in the ICU. Favorable outcome (which included those who were transferred to the ward) was seen in $65 \%$ of patients from ICU [Table 1].

\section{QT interval data}

The machine QTc value of the ECGs was used for outcome associations. There were total of 174 machine values which were available from day 1 . In the remaining 5 , it was not reported due to poor ECG tracings.

The mean QTc on day 1 for the total population was $445.2(S D=47.8)$. Mean QTc for females was $450.2(\mathrm{SD}=48.7)$. Mean QTc for males was $440.5(\mathrm{SD}=46.7)$ [Table 2].

Number of patients with prolonged QTc on day $1=52(30 \%)$.

Among the males, 24 (27\%) had prolonged QTc. Among the females, $28(33 \%)$ had prolonged QTc.

Analyzing the QTc in the various age groups the mean QTc was longest in the $40-60$ age group, $n=56$, was $448.25(\mathrm{SD}=53.4)$.

On reviewing the medications that patients were on prior to ICU admission, 2 were on HCQ and 1 on phenytoin, which are known to affect the QT interval. Of these only the patient on phenytoin had a prolonged QTC on day 1 . The no of patients with QTc $>500=26(14.9 \%)$.

QTc intervals of the ECGs of day 3 of admission (here QTc of ECGs was available for 109 patients and for the others the machine values were not available).

The mean QTc interval on day $3=436.61$. The mean for the males $=438.03$ and for the females $=436.61$.

The percentage of patients with prolonged QTc $=19.3 \%$. The percentage of males with prolonged QTc $=15.7 \%$. The percentage of Females with prolonged QTC $=22.4 \%$.

As seen from Table 3 the patients had a wide range of primary diagnosis admitted into the medical ICU. The most common was infection, followed by cardiac related problems. Often these patients had multiple organ involvements.

\section{Comparison of day 1 and day 3 corrected QT interval}

The prevalence of QTc prolongation dropped from $30 \%$ on day $1-19 \%$ on day 3 . There was a reduction in the mean QTc in from 445.2 to 436.6 [Table 2]. The incidence of new prolonged QTc on day 3 was seen in only 9 patients. From the data, it is seen that the mean QTc and the number of patients with prolonged QTC have reduced between day 1 and day 3 . This value was found to be statistically significant $(P=0.011)$. 


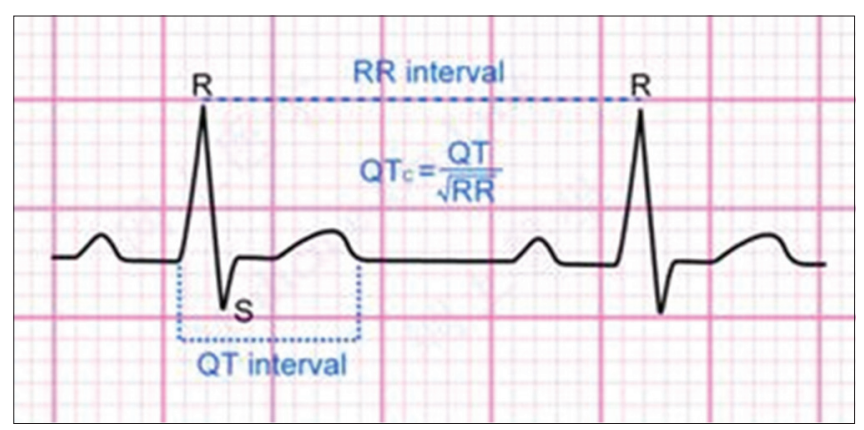

Figure I: The QT, RR interval, and Bazett's formula

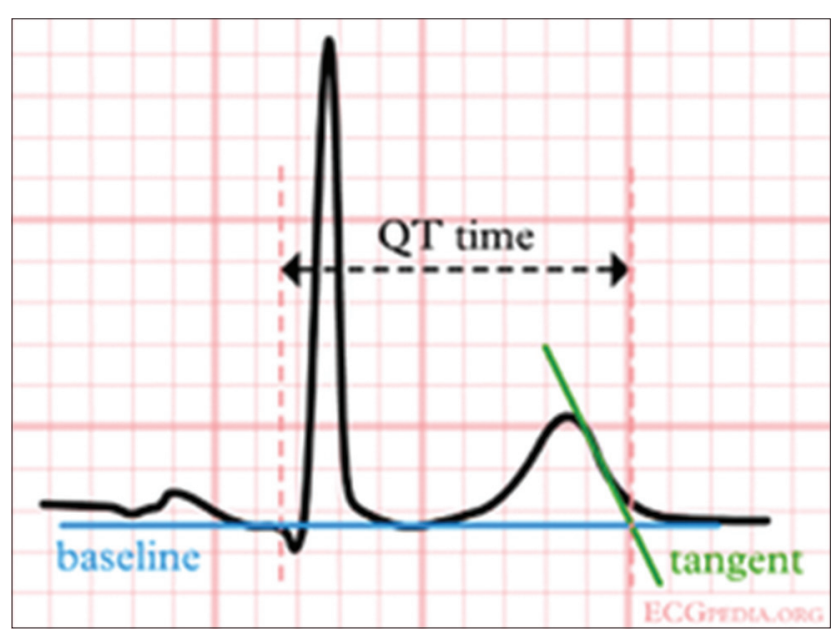

Figure 2: Identifying the end of the $T$ wave ${ }^{[6,1]]}$

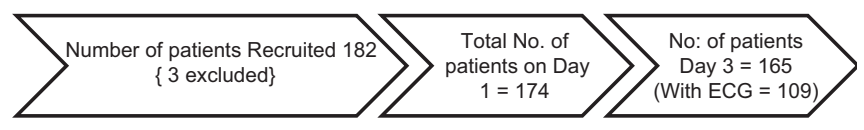

Figure 3: Flowchart of patients

\section{Comparison of machine and manual corrected $Q T$ interval}

Comparing QTc calculated by investigators. Machine-measure of agreement ICC $=89 \%$. Furthermore, comparing QTc calculated by cardiologist versus investigator. Measure of agreement ICC $=84 \%$.

\section{Analysis of the QT prolonging drugs in the study}

In this study, 35\% (61) of all patients received drugs with QT prolonging action. Of patients with a prolonged QTc at admission $18(35 \%)$ received a QT prolonging drug. These drugs included azithromycin, ondansetron, levofloxacin, amiodarone, haloperidol, and fluconazole. QTc prolonging drugs given in the ICU [Figure 4].

\section{Patient outcomes}

A favorable outcome was - discharge from ICU and hospital while an adverse was defined as death or discharged against medical advice.

\begin{tabular}{lc}
\hline Table I: Baseline data of admitted patients & \\
\hline Characteristic & Number $(\%)$ \\
\hline Age & $45.4(\mathrm{SD}=\mid 7.9)$ \\
Females & $85(49)$ \\
Males & $89(5 \mathrm{I})$ \\
Admitting reason* & \\
$\quad$ Respiratory distress & $\mathrm{II}(42)$ \\
Hemodynamic compromise & $86(3 \mathrm{I})$ \\
Neurology & $44(\mathrm{I})$ \\
Poisoning & $30(1 \mathrm{I})$ \\
Scores & \\
SOFA & $8 . \mathrm{I}(\mathrm{SD}=4.46)$ \\
APACHE II & $23.3(\mathrm{SD}=8.2)$ \\
Mean duration of stay & \\
$\quad$ ICU & 7 days $(\mathrm{SD}=5.8)$ \\
$\quad$ Hospital & I3 days $(\mathrm{SD}=10 . \mathrm{I})$ \\
Adverse outcome from ICU & $6 \mathrm{I}(35)$ \\
\hline
\end{tabular}

*Some patients had more than one reason for admission into ICU. SD: Standard deviation; SOFA: Sequential organ failure assessment; APACHE: Acute physiology and chronic health evaluation; ICU: Intensive care unit

On comparing those with normal and prolonged QTC and the outcome from ICU and hospital [Table 4] in the normal QT group $25 \%$ and $32 \%$ had an adverse outcome in the ICU and hospital respectively versus $52 \%$ in the prolonged QT group, respectively. The Odds ratio for an adverse event in the patients with a prolonged QTC was 3.17 (CI: 1.52-6.63) $(P=0.001)$ in ICU and 2.27 (CI: 1.11-4.66) $(P=0.014)$ for the hospital stay. The duration of ICU stay was not different 7.2 days, in the normal QTC group versus 7.3 days in the prolonged QTc group.

Ventilator-free days is a representation of the duration of ventilation in the ICU and assesses therapies in an ICU. It combines the effect of duration of ventilation, morbidity, and mortality. VFD = number of days where the patient breathed without ventilation $(28-\mathrm{X})$. If the patient dies or is ventilated over 28 days, the value is 0 . On assessing the ventilator-free days, the normal QT group had a longer period 18.3 versus 11.5 implying a better outcome [Figure 5].

Among the monitored events, there was a trend toward increased number of supraventricular tachycardia, premature ventricular complexes, ventricular tachycardia, and fibrillation in the prolonged QT group, but the numbers were not statistically significant [Table 5].

\section{Discussion}

This to our knowledge is the first Indian study to analyze the QTc interval in patients who are acutely ill in a medical ICU. This was a population with varying illnesses and age groups who have chronic as well as acquired risk factors, which are potentially correctible. Here the mean age of the patients was 45 years and this is in contrast to ICUs in the west where more population are 


\begin{tabular}{lcc}
\hline \multicolumn{3}{l}{ Table 2: Comparison of QT intervals of day I and day 3} \\
\hline Characteristic & Day I total = I 74 & Day 3 total $=109$ \\
\hline Prolonged QTc $(\%)$ & $52 / I 74(30)$ & $2 \mathrm{I} / \mathrm{I09}(\mathrm{I} 9)$ \\
Males & $24 / 89(27)$ & $8 / 5 \mathrm{I}(\mathrm{I})$ \\
Females & $28 / 85(33)$ & $13 / 58(22)$ \\
Mean QTc & $445.2(\mathrm{SD}=47.8)$ & $436.6 \mathrm{I}(\mathrm{SD}=45.16)$ \\
Males & $440.5(\mathrm{SD}=46.7)$ & $438.03(\mathrm{SD}=45.28)$ \\
Females & $450.2(\mathrm{SD}=48.7)$ & $436.6 \mathrm{I}(\mathrm{SD}=45.4)$ \\
\hline
\end{tabular}

SD: Standard deviation; QTc: Corrected QT interval

Table 3: Primary diagnosis and QTc interval

\begin{tabular}{lccc}
\hline Diagnosis & Total number & Prolonged QT & Mean QT (SD) \\
\hline Cardiac & 33 & 15 & $456.8(65 . I)$ \\
Neurological & 17 & 5 & $441.2(34.5)$ \\
Respiratory & 30 & 8 & $439.8(45.6)$ \\
Infection & 52 & 13 & $44 I .5(4 I .1)$ \\
Poisoning & 30 & 7 & $432.1(38.1)$ \\
Miscellaneous & 12 & 4 & $459.8(62.4)$ \\
Total & 174 & 52 & $455.2(47.8)$ \\
\hline
\end{tabular}

Cardiac: Diagnosis included acute coronary syndromes (NSTEMI and unstable angina) and cardiac failure; Neurological causes included: Hanging (partial and complete), heat-related illnesses and benzodiazepine related altered sensorium and TB meningitis; Respiratory: Pneumonia, pleural effusion, malignancy, COPD, and asthma exacerbations; Infection: Undifferentiated febrile illness, febrile neutropenia, scrub typhus, pyelonephritis, cholangitis, and infections in chronic inflammatory diseases; Poisonings included: Organophosphorus, alprazolam, olanzapine, oleander, and oduvanthalai poisoning; Miscellaneous: Pancreatitis, flare of inflammatory diseases like SLE, DKA. SD: Standard deviation; QTc: Corrected QT interval; NSTEMI: Non-ST segment elevation MI; TB: Tuberculous; COPD: Chronic obstructive pulmonary disease; SLE: Systemic lupus erythematosus; DKA: Diabetic ketoacidosis

of older age. ${ }^{[15]}$ There was an equal gender distribution, consisting of $49 \%$ females the patients were also quite morbid with a mean APACHE $=23.3(\mathrm{SD}=8.2)$. There were a higher proportion of patients with infections and poisonings.

In our study, almost one-third of the population had a prolonged QTC on day 1 of admission. Other studies have also shown similar trends in the prevalence of a prolonged QTc in ICUs [Table 6]. In a study by Pickham et al it was $24 \%$, Kozik et al $52 \%$ and Tisdale $28 \%$. $6,7,16]$ From the data, it is seen that the mean QTc and the number of patients with prolonged QTc had reduced between day 1 and day $3(30-19 \%)$. This value was found to be statistically significant $(P=0.011)$.

The high prevalence is possibly due to the acquired risk factors for a QTc prolongation in a newly admitted critically ill patient. The resolution is possibly explained by the fact that after 2 days in ICU the patient is stabilized with less biochemical derangements and control of the underlying acute illness.

It is found that among patients with a prolonged QTC at admission $18(35 \%)$ received a QT prolonging drug. In other studies by Tisdale et al., $34.7 \%$ of the population with a prolonged QTc received a QT prolonging drug. ${ }^{[7]}$ It was noted that an additional QT prolongation of $>60$ ms occurred in $57.1 \%$ of these patients. The study by Kozik and Wung noted that 59\% received a drug with known QT prolonging action. ${ }^{[16]}$ In this study, the potential QT prolonging drugs used were azithromycin, ondansetron, levofloxacin, amiodarone, haloperidol, and fluconazole.

Assessing the association with QTc and outcomes we found that the patients with a prolonged QTc had three times the odds of an adverse outcome in the ICU than those with a normal QTc at admission. This was also represented in the hospital stay adverse outcome and number of ventilator-free days.

This was similarly noted in a study by Haugaa et al. and Pickham et al. ${ }^{[5,6]}$

Even in patients with organophosphorous poisoning in a medical ICU, conducted by Shadnia et al the period of hospitatlisation and mortality rate was higher in the prolonged QTc group. ${ }^{[17]}$

Although bradycardia can cause a prolongation in QTc, in Table 5 we note that patients in both groups had tachycardia, but we see that the population in the group with QTc prolongation had a slower heart rate. Though this may be statistically significant, it cannot be extrapolated to clinical significance.

We did a univariate analysis to determine any predictors of a prolonged QTc particularly age, sex, admission diagnosis, history of stroke, cardiac illness, and biochemical abnormalities (potassium, calcium, magnesium, and creatinine). We did not find any strong associations.

We also performed a logistic regression for predictors of adverse outcomes in ICU using similar variables along with admission diagnosis and the SOFA and APACHE scores. The significant variables were the QTc and the composite scoring systems.

This study, being observational in nature, does not attribute a causative effect of a prolonged QTc and an adverse outcome but indicates a strong association. It was not powered to determine the potential predictors of a prolonged QTC or adverse outcome.

The QT analysis employs a simple ECG from which the QT interval can be calculated with simple instructions. 


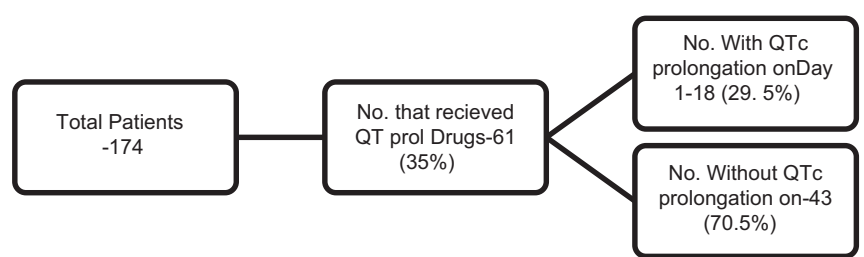

Figure 4: Corrected QT interval prolonging drugs given in the Intensive Care Unit

\begin{tabular}{lccccccc}
\hline \multicolumn{2}{l}{ Table 4: Comparison of outcomes based on QTc } \\
\hline QTc & \multicolumn{2}{c}{ ICU outcome } & & \multicolumn{2}{c}{ Hospital outcome } & Total \\
\cline { 2 - 3 } & Favorable & Adverse & & Favorable & Adverse & \\
\hline Normal & 91 & $3 I$ & & 83 & 39 & 122 \\
Prolonged & 25 & 27 & & 25 & 27 & 52 \\
Total & 116 & 58 & & 108 & 66 & 174 \\
\hline
\end{tabular}

QTc: Corrected QT interval; ICU: Intensive care unit

Table 5: Demographic and clinical variable differences between those with normal and prolonged QTc

\begin{tabular}{|c|c|c|c|}
\hline \multirow[t]{2}{*}{ Predictors (n) } & $\begin{array}{l}\text { QT normal-n (\%) } \\
\text { or mean } \pm \text { SD }(n)\end{array}$ & $\begin{array}{l}\mathrm{QT} \text { prolonged- } n(\%) \\
\text { or mean } \pm \mathrm{SD}(n)\end{array}$ & \multirow[t]{2}{*}{$P$} \\
\hline & Total $=\mid 22$ & Total $=52$ & \\
\hline Age (174) & $44.6(122)$ & $46.8(52)$ & 0.429 \\
\hline Male (89) & $65(73)$ & $24(27)$ & 0.390 \\
\hline Female (85) & $57(67)$ & $28(33)$ & 0.389 \\
\hline \multicolumn{4}{|l|}{ Reason for admission } \\
\hline Respiratory & 80 & 39 & 0.221 \\
\hline Hemodynamic & 59 & 27 & 0.667 \\
\hline Neurological & 33 & II & 0.413 \\
\hline Poisoning & 23 & 7 & 0.389 \\
\hline \multicolumn{4}{|l|}{ Clinical history (\%) } \\
\hline Diabetes (58) & $44(76)$ & $14(24)$ & 0.028 \\
\hline Hypertension (57) & $39(68)$ & $18(32)$ & 0.544 \\
\hline IHD (I8) & $12(67)$ & $6(33)$ & 0.172 \\
\hline Old CVA (II) & $9(8 \mathrm{I})$ & $2(19)$ & 0.516 \\
\hline Renal failure (18) & $7(39)$ & II (6I) & 1.000 \\
\hline \multicolumn{4}{|l|}{ Lab parameters } \\
\hline Sodium (169) & $134.7 \pm 15.6(118)$ & $\mid 34.9 \pm 8.9(5 \mid)$ & 0.918 \\
\hline Potassium (I7I) & $4.0 \pm$ I (I19) & $3.8 \pm$ I (52) & 0.152 \\
\hline Calcium (147) & $4.3 \pm 1.2(103)$ & $4.1 \pm 1.0(42)$ & 0.377 \\
\hline Magnesium (52) & $2.4 \pm 1.6(42)$ & $2.4 \pm 1.1(10)$ & 0.971 \\
\hline Creatinine (I7I) & $2.1 \pm 1.9(121)$ & $2.6 \pm 3.2(50)$ & 0.376 \\
\hline Glucose (158) & $142.4 \pm(\mid 13)$ & $149.4 \pm(45)$ & 0.555 \\
\hline $\operatorname{ACS}(13)$ & 8 & 5 & 0.483 \\
\hline Arrhythmias (33) & 22 & 11 & 0.631 \\
\hline HR on ECG $(n=150)$ & $119 \pm 31(106)$ & $109 \pm 26(44)$ & 0.050 \\
\hline QTc & $419.1(S D=19.9)$ & $506.6(\mathrm{SD}=36.4)$ & 0.000 \\
\hline
\end{tabular}

Arrhythmias: Supraventricular tachycardia, ventricular tachycardia, ventricular fibrillation, premature ventricular contraction, atrial fibrillation, heart blocks, sudden cardiac arrest, and Torsades de pointes. SD: Standard deviation; ACS: Acute coronary syndrome; IHD: Ischemic heart disease; QTc: Corrected QT interval; HR: Heart rate; ECG: Electrocardiography; CVA: Cerebrovascular accident

Table 6: Prevalence of prolonged QTc in different studies

\begin{tabular}{lcccc}
\hline Study & Our study & Pickham et al. $^{\left[{ }^{[6]}\right.}$ & Kozik $^{[14]}$ & Tisdale et al. ${ }^{[7]}$ \\
\hline QTcprevalence $(\%)$ & 30 & 24 & 52 & 28 \\
\hline
\end{tabular}

QTc: Corrected QT interval

The correlation with the machine and the calculated value implies that this can also be used in a standardized machine. Considering that QTc is an index of severity

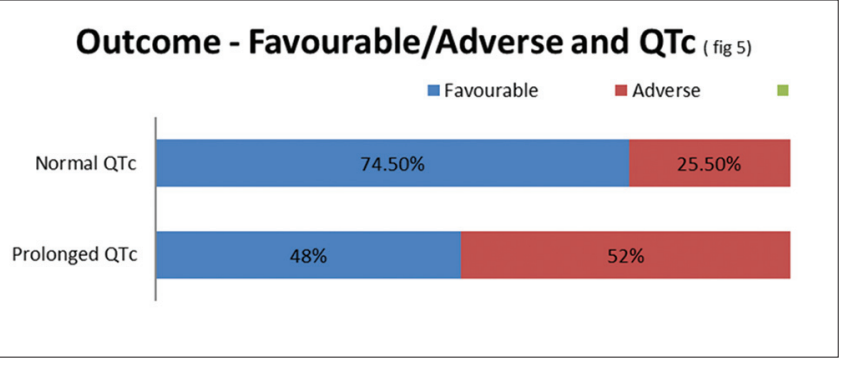

Figure 5: Outcome - Favorable/adverse and corrected QT interval

of the underlying illness and a predictor of adverse ICU outcomes, it can be incorporated into predictive diagnostic scores.

\section{Limitations}

This was limited to a single center experience and the patient profile compared to western data consisted of more morbid patients. QT variations can be transient and continuous ECG monitoring was not possible, hence we could have missed out other prolonged QT intervals or arrhythmias. A longer follow-up period in the ICU could have revealed a clearer trend to the QT intervals in relation to the variables analyzed. We did not have a baseline ECG or an ECG at discharge which would demonstrate premorbid values.

\section{Conclusions}

This study demonstrates the high prevalence (30\%) of a prolonged QTC in patients at admission in a Medical ICU. It was noted that $35 \%$ of these patients received a drug that could further prolong the interval. Acutely ill patients with prolonged QT intervals had nearly a three times odds ratio for an adverse event in the ICU, twice for the hospital stay. A simple ECG and a calculated QT interval can be used to plan management and caution us on probable electrolyte abnormalities and drug therapies. It can even be incorporated as a tool for prognostication of patients in a medical ICU.

\section{Financial support and sponsorship}

Nil.

\section{Conflict of interest}

There are no conflict of interest.

\section{References}

1. Goldhill DR, Sumner A. Outcome of intensive care patients in a group of British intensive care units. Crit Care Med 1998;26:1337-45.

2. Knaus WA, Draper EA, Wagner DP, Zimmerman JE. APACHE II: A severity of disease classification system. Crit Care Med 1985;13:818-29.

3. Ferreira FL, Bota DP, Bross A, Mélot C, Vincent JL. Serial evaluation of the SOFA score to predict outcome in eritically ill patients. JAMA 2001;286:1754-8. 
4. Le Gall JR, Lemeshow S, Saulnier F. A new Simplified Acute Physiology Score (SAPS II) based on a European/North American multicenter study. JAMA 1993;270:2957-63.

5. Haugaa KH, Bos JM, Tarrell RF, Morlan BW, Caraballo PJ, Ackerman MJ. Institution-wide QT alert system identifies patients with a high risk of mortality. Mayo Clin Proc 2013;88:315-25.

6. Pickham D, Helfenbein E, Shinn JA, Chan G, Funk M, Weinacker A, et al. High prevalence of corrected QT interval prolongation in acutely ill patients is associated with mortality: Results of the QT in Practice (QTIP) Study. Crit Care Med 2012;40:394-9.

7. Tisdale JE, Wroblewski HA, Overholser BR, Kingery JR, Trujillo TN, Kovacs RJ. Prevalence of QT interval prolongation in patients admitted to cardiac care units and frequency of subsequent administration of $\mathrm{QT}$ interval-prolonging drugs: A prospective, observational study in a large urban academic medical center in the US. Drug Saf 2012;35:459-70.

8. Mabasa VH, Yokoyama S, Man D, Martyn J. Analysis of Orders for QTe-Prolonging Medication for Intensive and Cardiac Care Unit Patients with Pre-existing QTe Prolongation (QTIPPP Study). Can J Hosp Pharm 2011;64:412-8.
9. Garson A Jr. How to measure the QT interval - What is normal? Am J Cardiol 1993;72:14B-16.

10. Goldenberg I, Moss AJ, Zareba W. QT interval: How to measure it and what is normal. J Cardiovasc Electrophysiol 2006;17:333-6.

11. Available from: http://www.medicine-on-line.com/html/ecg/e0001en_ files/05.htm.

12. Available from: http://www.en.ecgpedia.org/wiki/File: LastigeQT1.png.

13. Moss AJ. Long QT syndrome. JAMA 2003;289:2041-4.

14. Al-Khatib SM, LaPointe NM, Kramer JM, Califf RM. What clinicians should know about the QT interval. JAMA 2003;289:2120-7.

15. Ng TM, Olsen KM, McCartan MA, Puumala SE, Speidel KM, Miller MA, et al. Drug-induced QTc-interval prolongation in the intensive care unit: Incidence and predictors. J Pharm Pract 2010;23:19-24.

16. Kozik TM, Wung SF. Acquired long QT syndrome: Frequency, onset, and risk factors in intensive care patients. Crit Care Nurse 2012;32:32-41.

17. Shadnia S, Okazi A, Akhlaghi N, Sasanian G, Abdollahi M. Prognostic value of long QT interval in acute and severe organophosphate poisoning. J Med Toxicol 2009;5:196-9. 